\title{
A NOTE ON EXTREMAL MAPPINGS OF FINITE DISTORTION
}

\author{
Stanislav Hencl, Pekka Koskela, and Jani Onninen
}

\section{Introduction}

We consider Sobolev mappings $f \in W_{\text {loc }}^{1,1}\left(\Omega, \mathbb{R}^{2}\right)$, where $\Omega$ is a subdomain of $\mathbb{R}^{2}$. Thus, for almost every $x \in \Omega$, we can speak of the linear transform $D f(x)$ : $\mathbb{R}^{2} \rightarrow \mathbb{R}^{2}$, called the differential of $f$ at the point $x$. The Jacobian determinant $J(x, f)$ is the determinant of the matrix $D f(x), J(x, f)=\operatorname{det} D f(x)$. We say that a mapping $f: \Omega \rightarrow \mathbb{R}^{2}$ has finite distortion if the following three conditions are satisfied:

(i) $f \in W_{\text {loc }}^{1,1}\left(\Omega, \mathbb{R}^{2}\right)$,

(ii) The Jacobian determinant $J(x, f)$ of $f$ is locally integrable,

(iii) There is a measurable function $K=K(x)$, finite almost everywhere, such that $f$ satisfies the distortion inequality

$$
|D f(x)|^{2} \leqslant K(x) J(x, f) \quad \text { a.e. } x \in \Omega .
$$

Above we used the operator norm of the differential matrix, defined by $|D f(x)|=$ $\sup \{|D f(x) h| ;|h|=1\}$. The smallest function $K(x)$ for which the distortion inequality (1) holds, is called the outer distortion function of $f$, and defined by

$$
K(x, f)= \begin{cases}\frac{|D f(x)|^{2}}{J(x, f)}, & \text { if } J(x, f)>0 \\ 1, & \text { otherwise. }\end{cases}
$$

The operator norm of the differential matrix at (2) has the disadvantage of being insufficiently regular to deal with variational equations. We therefore introduce the outer distortion function

$$
\mathbb{K}(x, f)= \begin{cases}\frac{\|D f(x)\|^{2}}{J(x, f)}, & \text { if } J(x, f)>0 \\ 1, & \text { otherwise. }\end{cases}
$$

Here $\|\mathbf{A}\|^{2}=\frac{1}{2} \operatorname{Tr}\left(\mathbf{A}^{T} \mathbf{A}\right)$ is the mean Hilbert-Schmidt norm.

Received June 24, 2004.

Hencl and Koskela were supported in part by the Academy of Finland. Onninen by the National Science Foundation grant DMS-0400611.

2000 Mathematics Subject Classification. Primary 30C65; Secondary 35J15, 35J70, 30C70.

Keywords. Extremal, Calculus of Variations. 
The study of the connection between extremal mappings of finite distortion and harmonic mappings was initiated in [1] and further investigated in [2]. Consider the class $\mathcal{F}=\mathcal{F}\left(\Omega, \Omega^{\prime}\right)$ of $W_{\text {loc }}^{1,1}\left(\Omega, \mathbb{R}^{2}\right)$-regular homeomorphisms $f: \bar{\Omega} \rightarrow \overline{\Omega^{\prime}}$ of finite distortion for which $\mathbb{K}(x, f)$ is integrable in $\Omega$. In this paper, we prove the following result.

Theorem 1.1. Let $\Omega$ be a convex planar domain and $f_{\circ} \in \mathcal{F}=\mathcal{F}\left(\Omega, \Omega^{\prime}\right)$. Then the minimization problem

$$
\min _{f \in \mathcal{F}} \int_{\Omega} \mathbb{K}(x, f) d x, \quad f=f_{\circ} \text { on } \partial \Omega
$$

has a unique solution. This extremal map is a $\mathscr{C}^{\infty}$-diffeomorphism whose inverse is harmonic in $\Omega^{\prime}$.

In [1], the above result is established for the smaller class $\mathcal{F} \cap W_{\text {loc }}^{1,2}\left(\Omega, \mathbb{R}^{2}\right)$. Our result shows that the problem has a unique minimizer in the full class $W_{\text {loc }}^{1,1}\left(\Omega, \Omega^{\prime}\right)$. The proof given in [1] was based on the natural Sobolev regularity $W_{\text {loc }}^{1,2}$-assumption. Roughly speaking, this is the minimal regularity assumption which guarantees that we can integrate by parts against the Jacobian determinant i.e. apply Stokes' theorem. In this light, it looks surprising that we can relax the Sobolev regularity assumption here.

\section{An Identity}

Theorem 2.1. Let $\Omega$ and $\Omega^{\prime}$ be planar domains. Suppose $f \in W_{\text {loc }}^{1,1}\left(\Omega, \Omega^{\prime}\right)$ is a homeomorphism of finite distortion with

$$
\int_{\Omega} \mathbb{K}(x, f) d x<\infty
$$

Then the inverse map $h: \Omega^{\prime} \rightarrow \Omega$ belongs to $W_{\text {loc }}^{1,2}\left(\Omega^{\prime}, \Omega\right)$ and we have

$$
\int_{\Omega^{\prime}}\|D h(y)\|^{2} d y=\int_{\Omega} \mathbb{K}(x, f) d x
$$

We will split the proof of Theorem 2.1 into two parts. First, we study the Sobolev regularity of the inverse mapping. In the second part we show that the main identity (6) is valid. Our approach is motivated by the recent results in $[6]$.

2.1. The inverse belongs to $W_{\text {loc }}^{1,2}\left(\Omega^{\prime}, \Omega\right)$. Fix a point $y_{\circ} \in \Omega^{\prime}$ and a positive radius $r$ such that $\mathbb{B}_{3 r}=\mathbb{B}\left(y_{0}, 3 r\right) \subset \Omega$. Our starting point is to show the auxiliary estimate

$$
\operatorname{diam} f^{-1}\left(\mathbb{B}_{r}\right) \leqslant C\left(\int_{f^{-1}\left(\mathbb{B}_{2 r}\right)} K(x, f) d x\right)^{\frac{1}{2}} .
$$

To do this, we fix a smooth function $v: \mathbb{R}^{2} \rightarrow \mathbb{R}$ which satisfies the properties 
- $|\nabla v(y)| \leqslant \frac{C}{r}$ for all $y$,

- $0 \leqslant v(y) \leqslant 1$ for all $y$,

- $v(y)=1$ in $\mathbb{B}_{r}$ and $v(y)=0$ in $\mathbb{R}^{2} \backslash \mathbb{B}_{2 r}$.

Since $f$ is a homeomorphism in the Sobolev class $W_{\text {loc }}^{1,1}\left(\Omega, \Omega^{\prime}\right)$, it is differentiable almost everywhere. This elegant result is due to Gehring and Lehto [5]. Applying the chain rule and the identity $|D f(x)|^{2}=K(x, f) J(x, f)$ we have

$$
\int_{\mathbb{A}} \frac{|\nabla(v \circ f)|^{2}}{K(\cdot, f)} \leqslant \int_{\mathbb{A}}|\nabla v(f(x))|^{2} J(x, f) d x,
$$

where $\mathbb{A}=f^{-1}\left(\mathbb{B}_{2 r}\right)$.

Because $f$ is a homeomorphism in the Sobolev class $W_{\text {loc }}^{1,1}\left(\Omega, \Omega^{\prime}\right)$, we can apply the change of variable formula and find that

$$
\int_{\mathbb{A}}|\nabla v(f(x))|^{2} J(x, f) d x \leqslant \int_{f(\mathbb{A})}|\nabla v(y)|^{2} d y .
$$

Notice that we claim only the inequality here. Actually, equality appears if $f$ preserves measure zero sets i.e. satisfies the condition $(N)$. This need not be the case in our setting. For the proof of (9) we refer to [7, Corollary 6.3.1]. Combining (8) and (9) with the estimate $|\nabla v(y)| \leqslant \frac{C}{r}$ we find that the integral

$$
\int_{\mathbb{A}} \frac{|\nabla(v \circ f)|^{2}}{K(\cdot, f)}
$$

is bounded above by a constant. For a lower bound we first notice that

$$
\operatorname{diam} f^{-1}\left(\mathbb{B}_{r}\right) \leqslant \int_{\mathbb{A}}|\nabla(v \circ f)| .
$$

Indeed, we may assume that $\operatorname{diam} f^{-1}\left(\mathbb{B}_{r}\right)=\alpha$ and that the set $f^{-1}\left(\mathbb{B}_{r}\right)$ contains the origin and the point $(0, \alpha)$. For a.e. $0<t<\alpha$, we have

$$
1 \leqslant \int_{\left\{x_{2}=t\right\}}|\nabla(v \circ f)| d x_{1},
$$

where by $\left\{x_{2}=t\right\}$ we mean a suitable line segment in $\mathbb{A}$, parallel to the $x_{1}$-axis. Integrating over $0<t<\alpha$ we obtain the desired inequality (11). Hence, Hölder's inequality gives us

$$
\operatorname{diam} f^{-1}\left(\mathbb{B}_{r}\right) \leqslant \int_{\mathbb{A}}|\nabla(v \circ f)| \leqslant\left(\int_{\mathbb{A}} \frac{|\nabla(v \circ f)|^{2}}{K(\cdot, f)}\right)^{\frac{1}{2}}\left(\int_{\mathbb{A}} K(\cdot, f)\right)^{\frac{1}{2}} .
$$

Now, the claim (7) follows from the boundedness of the integral (10).

Next, we will first prove that $f^{-1}$ is ACL i.e. absolutely continuous on almost all lines parallel to the coordinate axes. Later, we will realize that the estimate which gives the ACL-property actually also shows that $f^{-1} \in W_{\mathrm{loc}}^{1,2}\left(\Omega^{\prime}, \Omega\right)$. For the ACL-property, fix an open cube $\mathbb{Q}$ such that $\overline{\mathbb{Q}} \subset \Omega^{\prime}$. It suffices to show that $f^{-1}$ is ACL in this cube. By symmetry, it suffices to consider line segments 
parallel to the $x_{2}$-axis. Assume that $\mathbb{Q}=\mathbb{I}_{1} \times \mathbb{I}_{2}$. Next, for each Borel set $\mathbb{E} \subset \mathbb{I}_{1}$ we define

$$
\eta(\mathbb{E})=\int_{f^{-1}\left(\mathbb{E} \times \mathbb{I}_{2}\right)} K(x, f) d x .
$$

By the Lebesgue differentiation theorem [11, Theorem 23.5], $\eta$ has a finite derivative $\eta^{\prime}(y)$ for almost every $y \in \mathbb{I}_{1}$. Fix such a $y$. We will prove that $f^{-1}$ is absolutely continuous on the segment $\{y\} \times \mathbb{I}_{2}$, which proves the claim. Let $\left\{\triangle_{i}\right\}_{i=1}^{\ell}, \triangle_{i}=\left[a_{i}, b_{i}\right]$, be a union of closed intervals on $\mathbb{I}_{2}$ whose interiors are mutually disjoint.

Fix a natural number $\beta$ such that

$$
\frac{1}{\beta}<\frac{\min \left\{\operatorname{dist}\left(\cup \Delta_{i}, \partial \mathbb{I}_{2}\right), b_{1}-a_{1}, \ldots, b_{\ell}-a_{\ell}\right\}}{17} .
$$

Now a standard covering argument [11, Lemma 31.1] gives us a number $\delta$ depending on $\beta$ such that for $0<r<\delta$ we have a covering $B_{1}^{i}, \ldots, B_{\kappa_{i}}^{i}$ of $\triangle_{i}$ which has the properties

- $\operatorname{diam}\left(B_{j}^{i}\right)=2 r$,

- at most 4 of the sets $B_{j}^{i}$ intersect at any point,

- $B_{j}^{i}$ is contained in the $\frac{1}{\beta}$-neighborhood of $\triangle_{i}$.

Pick the balls $\mathbb{B}_{j}^{i}=\mathbb{B}\left(x_{j}^{i}, r\right)$ centered in our segment with $\mathbb{B}_{j}^{i} \cap\{y\} \times \mathbb{I}_{2}=\{y\} \times B_{j}^{i}$. To shorten our notation we write $\mathbb{A}_{j}^{i}=f^{-1} \mathbb{B}\left(x_{j}^{i}, 2 r\right)$. Now, applying the estimate (7) and Hölder's inequality we have

$$
\begin{aligned}
\sum_{i=1}^{\ell}\left|f^{-1}\left(y, a_{i}\right)-f^{-1}\left(y, b_{i}\right)\right| & \leqslant \sum_{i=1}^{\ell} \sum_{j=1}^{\kappa_{i}} \operatorname{diam}\left(f^{-1} \mathbb{B}_{j}^{i}\right) \\
& \leqslant C \sum_{i=1}^{\ell} \sum_{j=1}^{\kappa_{i}}\left(\int_{\mathbb{A}_{j}^{i}} K(x, f) d x\right)^{\frac{1}{2}} \\
& =C \sum_{i=1}^{\ell} \sum_{j=1}^{\kappa_{i}} r^{\frac{1}{2}}\left(\frac{1}{r} \int_{\mathbb{A}_{j}^{i}} K(x, f) d x\right)^{\frac{1}{2}} \\
& \leqslant C\left(\sum_{i=1}^{\ell} \sum_{j=1}^{\kappa_{i}} r\right)^{\frac{1}{2}}\left(\frac{1}{r} \sum_{i=1}^{\ell} \sum_{j=1}^{\kappa_{i}} \int_{\mathbb{A}_{j}^{i}} K(x, f) d x\right)^{\frac{1}{2}} .
\end{aligned}
$$

From the geometry of our covering it follows that the overlapping of $\mathbb{A}_{j}^{i}$ is at most 14. Therefore, we have

$$
\frac{1}{r} \sum_{i=1}^{\ell} \sum_{j=1}^{\kappa_{i}} \int_{\mathbb{A}_{j}^{i}} K(x, f) d x \leqslant 14 \frac{\eta((y-2 r, y+2 r))}{r}
$$


and

$$
\sum_{i=1}^{\ell} \sum_{j=1}^{\kappa_{i}} r \leqslant 14\left|\bigcup_{i=1}^{\ell}\left[a_{i}-1 / \beta, b_{i}+1 / \beta\right]\right|
$$

By taking the upper limit when $r \rightarrow 0$ and then letting $\beta \rightarrow \infty$, we conclude that

$$
\left(\sum_{i=1}^{\ell}\left|f^{-1}\left(y, a_{i}\right)-f^{-1}\left(y, b_{i}\right)\right|\right)^{2} \leq C \eta^{\prime}(y) \sum_{i=1}^{\ell}\left(b_{i}-a_{i}\right)
$$

which proves that $f^{-1}$ is absolutely continuous on the line segment $y \times \mathbb{I}_{2}$, as desired. The full claim follows because it is proven in [9, Lemma 2.17] that the estimate (17) guarantees that $f^{-1} \in W_{\text {loc }}^{1,2}\left(\Omega^{\prime}, \Omega\right)$.

2.2. The main identity. It is well-known [4], [10, Corollary 1, p.182] that a planar homeomorphism of the Sobolev class $W^{1,2}$ satisfies the condition $(N)$. The condition $(N)$ means that a mapping maps sets of measure zero to sets of measure zero. Therefore, $\mathbb{K}(h(\cdot), f) J(\cdot, h) \in L_{l o c}^{1}\left(\Omega^{\prime}\right)$ and for all $\mathbb{B}_{3 r} \subset \Omega^{\prime}$ we have

$$
\int_{h\left(\mathbb{B}_{2 r}\right)} \mathbb{K}(x, f) d x=\int_{\mathbb{B}_{2 r}} \mathbb{K}(h(y), f) J(y, h) d y .
$$

Since $h \in W_{\text {loc }}^{1,2}\left(\Omega^{\prime}, \Omega\right)$, the mapping $h$ is differentiable almost everywhere [5]. This together with (7) and (18) implies that

$$
\|D h(y)\|^{2} \leq C \mathbb{K}(h(y), f) J(y, h) \quad \text { a.e. }
$$

Therefore, the measure of the set

$$
\mathbb{O}=\left\{y \in \Omega^{\prime} ; \quad J(y, h)>0 \text { and } h \text { is differentiable at } y\right\}
$$

equals $\left|\left\{y \in \Omega^{\prime} ; D h(y) \neq 0\right\}\right|$. First, the change of variable for $f$ gives

$$
\int_{\Omega^{\prime}}\|D h(y)\|^{2} d y \geqslant \int_{\Omega}\|D h(f(x))\|^{2} J(x, f) d x .
$$

By [8, Theorem 1.2.], the Jacobian determinant of $f$ is strictly positive almost everywhere and we conclude from the definition of $\mathbb{K}$, and the identities (see e.g. $[1$, Section 3])

$$
\|B\|=\left\|B^{T}\right\|,\left(A^{T}\right)^{-1} \operatorname{det} A=\operatorname{adj} A,\|\operatorname{adj} A\|=\|A\|,
$$

that

$$
\int_{\Omega^{\prime}}\|D h(y)\|^{2} d y \geqslant \int_{\Omega} \mathbb{K}(x, f) d x
$$


On the other hand, the change of variable formula for $h$ together with the $(N)$ condition for $h$ and the definition of $\mathbb{O}$ gives

$$
\begin{aligned}
\int_{\Omega^{\prime}}\|D h(y)\|^{2} d y & =\int_{\mathbb{O}}\|D h(y)\|^{2} d y \\
& =\int_{h(\mathbb{O})}\|D h(f(x))\|^{2} J(x, f) d x \\
& \leqslant \int_{\Omega} \mathbb{K}(x, f) d x .
\end{aligned}
$$

The desired identity follows.

\section{Proof of the main theorem}

After having the main identity, the claim follows as in [1]. For the convenience of the reader we give the argument also here. Let

$$
\mathcal{H}=\mathcal{H}\left(\Omega^{\prime}, \Omega\right)=\left\{h=f^{-1}: \overline{\Omega^{\prime}} \rightarrow \bar{\Omega}: f \in \mathcal{F}\left(\Omega, \Omega^{\prime}\right)\right\} .
$$

In light of the main identity (6), it is equivalent to minimize the Dirichlet problem:

$$
\inf _{h \in \mathcal{H}} \int_{\Omega^{\prime}}\|D h\|^{2}, \quad h=h_{\circ} \text { on } \partial \Omega^{\prime} .
$$

The existence of solutions in $h_{\circ}+W_{\circ}^{1,2}\left(\Omega^{\prime}, \mathbb{R}^{2}\right)$ to this well-known variational problem is guaranteed by the principles of convex analysis. The uniqueness of the solution follows from the strict convexity of the functional $\|\cdot\|^{2}$. The only point to make is that such a minimizer lies in $\mathcal{H}\left(\Omega^{\prime}, \Omega\right)$. This follows from the fundamental theorem of Radó, Kneser and Choquet, see [3]: if $\Omega \subset \mathbb{R}^{2}$ is a bounded convex domain, then each homeomorphism $h_{\circ}: \partial \Omega^{\prime} \rightarrow \partial \Omega$ has a unique continuous extension $h: \overline{\Omega^{\prime}} \rightarrow \bar{\Omega}$ which is a univalent harmonic map with a non-vanishing Jacobian. Then $f=h^{-1}$ is a homeomorphism of finite distortion. This extension $h$ is also a solution to the variational problem, and by uniqueness of the minimizer, this extension is the minimizer. Its inverse $f$ is a real analytic diffeomorphism which minimizes (4) by the main identity.

\section{References}

[1] K. Astala, T. Iwaniec, G. J. Martin, and J. Onninen, Extremal mappings of finite distortion. Proc. London Math. Soc., to appear.

[2] K. Astala, T. Iwaniec, and G. J. Martin, Deformations with smallest mean distortion and the nitsche conjecture. Preprint.

[3] P. Duren, Harmonic mappings between planar domains. Cambridge University Press, 2004.

[4] F. W. Gehring, Definitions and exceptional sets for quasiconformal mappings. Ann. Acad. Sci. Fenn. Ser. A I 281 (1960), 1-28.

[5] F. W.Gehring, and O. Lehto, On the total differentiability of functions of a complex variable. Ann. Acad. Sci. Fenn. Ser. A I 272 (1959), 1-9. 
[6] S. Hencl, and P. Koskela, Regularity of the inverse of a planar Sobolev homeomorphism. Preprint.

[7] T. Iwaniec, and G. J. Martin, Geometric Function Theory and Non-linear Analysis. Oxford Mathematical Monographs. 2001.

[8] P. Koskela, and J. Malý, Mappings of finite distortion: the zero set of the Jacobian. J. Eur. Math. Soc. (JEMS) 5 (2003), 95-105.

[9] O. Martio, S. Rickman, and J. Väisälä, Definitions for quasiregular mappings. Ann. Acad. Sci. Fenn. Ser. A I Math. (1969) 1-40.

[10] Yu. G. Reshetnyak, Space mappings with bounded distortion. American Mathematical Society. 1989.

[11] J. Väisälä, Lectures on n-dimensional quasiconformal mappings. Lecture Notes in Mathematics, Vol. 229. 1971, Springer-Verlag, Berlin-New York.

Department of Mathematics and Statistics, University of Jyväskylä, P.O. Box 35, Fin-40014, JyvÄSKYLÄ, Finland.

E-mail address: hencl@maths.jyu.fi

Department of Mathematics and Statistics, University of Jyväskylä, P.O. Box 35, Fin-40014, JYVÄSKYLÄ, Finland.

E-mail address: pkoskela@maths.jyu.fi

Department of Mathematics, University of Michigan, 525 E. University, Ann ArBOR, MI 48109, USA.

E-mail address: jonninen@umich.edu 